\title{
Proceedings for Lunch and Learn: Making science fun and exciting through social media
}

38th International Conference on High Energy Physics

3-10 August 2016

Chicago, USA

\section{Lauren Biron1}

Fermilab

PO Box 500

Batavia IL 60510-5011

United States of America

E-mail:lbiron@fnal.gov

\section{Julie Haffner2}

CERN

385 Route de Meyrin

1217 Meyrin

Switzerland

E-mail:julie.haffner@cern.ch

\author{
Clara Nellist 3 \\ IN $2 P 3$ \\ 3 rue Michel-Ange \\ 75794 Paris CEDEX 16 \\ France \\ E-mail: clara.nellist@cern.ch
}

\section{Dianna Cowern4 \\ http://physicsgirl.org/}

\author{
Arnaud Marsollier \\ CERN \\ 385 Route de Meyrin \\ 1217 Meyrin \\ Switzerland \\ E-mail:arnaud.marsollier@cern.ch
}

\begin{abstract}
Social media channels are vital for outreach and offer huge opportunities for scientists to directly engage with the public using nontraditional methods - including lots of creativity and humor. The physics community's presence is growing more significant, and this session (designed for early career researchers) provided a lively discussion with experts in the domain. We covered how to best use social media to raise public awareness of science, share excitement and progress, and cultivate support from followers. We also discussed some of the thornier issues in social media, such as capturing the complexity of both the scientific process and the science itself.
\end{abstract}




\section{Introduction}

Social media is a relatively new way of delivering news, but also a very immediate and sometimes intimate way of posting and sharing material, whether words, images, or video. It grants the ability to have a conversation in near real-time. Social media includes (but is not limited to) platforms such as Twitter, Facebook, LinkedIn, YouTube, Google+, Periscope, Snapchat, Tumblr, Flickr, and others.

This lunch session was presented by Lauren Biron of Fermilab, Clara Nellist of the ATLAS Experiment and IN2P3, Dianna Cowern of the physics YouTube series Physics Girl, and Julie Haffner of CERN.

\section{Overview}

Social media channels are vital for outreach and offer huge opportunities for scientists to directly engage with the public using nontraditional methods - including lots of creativity and humor. The physics community's presence is growing more significant, and this session (designed for early career researchers) provided a lively discussion with experts in the domain. We covered how to best use social media to raise public awareness of science, share excitement and progress, and cultivate support from followers. We also discussed some of the thornier issues in social media, such as capturing the complexity of both the scientific process and the science itself.

\section{Lauren Biron}

Because social media is informal, you can use it as an opportunity to show personality and share things in different ways that will appeal to different people. As a communicator for a science organization, one of the most important things to consider when posting content is "does it resonate with you in some way?" You are a member of the science-interested public - so you are essentially your own target audience. Scientists can also provide insight into aspects of science the public does not normally get to see - the process, the journey, and the fact that scientists are real people with diverse interests and backgrounds, not a monolithic other. Humanize the science and the people behind it to break down stigmas, stereotypes, and preconceptions about research.

Social media has many other uses, including maintaining an official presence for news, doing damage control, connecting with people, or even just providing incremental updates for really ardent fans. Regardless of what you use it for, remember to know your platform and use its features (whether that's hashtags, gifs, etc.). Not every tweet can have an Arrested Development gif, but if you spice up the more serious communication with some levity and entertainment, people are more likely to stick around and come across your other messages.

\section{Clara Nellist}

Communicating science and making it fun through a personal social media account and through one for a collaboration requires different approaches. For the former, I want to show a female physicist working, and allow people following me to see the person as well as the work; this inevitably leads to many photos of myself. However, there are ways to make science fun on social media without requiring selfies in a hard-hat. For example, an object that is well-known to many people, such as a dishwasher, that is used in a slightly different way for science can resonate with 
your audience.

When using social media on behalf of a collaboration, it is important to make it accessible to people who cannot come and visit in person. One example is the ATLAS Experiment's use of a 360-degree photograph of the ATLAS cavern, shared through Facebook. This is a more immersive experience than a static photograph, since the person viewing on a smartphone can move the screen to reveal a new part of the cavern. Another method is live interviews with physicists attending a conference (in this case ICHEP), which can be shared on Facebook. It is important to get results across, but we also want to show the physicists who do it.

A relatively new platform for the ATLAS Experiment was Instagram, the photo-sharing platform. Some people think that art and science are not related, but I do not agree. I think it is important to show the beauty in our work.

\section{Dianna Cowern}

Doing research is rewarding and a unique experience, but communicating it to the public with passion is also fun! Sometimes, it draws scientists away from their original path to focus on sharing science with those who may not understand the process (or the results). Enthusiasm is one of the most crucial parts of science communication. If you are passionate about something and it makes you excited, that's something you should not try to hide in your presentation. Reading facts in a deadpan voice doesn't get anyone amped up about magnets - but showing how you can build a racetrack out of an AA battery and some copper coils and being legitimately excited about it certainly does!

Another key aspect is picking a platform that works well for you. In my case, it is YouTube videos, because I enjoy the scripting, filming and editing aspects. But you don't have to force it. Videos won't work for everyone, and they don't have to. If you prefer communicating on Twitter, or through blogs, you should do that instead. It's important to pick something you think you can stick with and fit into your busy schedule. So pick a process as well as a topic that you are enthusiastic about, and see if it's something you can keep up.

\section{Julie Haffner}

Physics can be fun, interactive, modern and accessible, and social media can help to prove it. "Begin a journey" is the watchword. You share information on social media to pique your followers' curiosity, raise their interest in science and encourage them to learn more. It's a way to foster engagement with the general public and give them the feeling that they are part of a community.

Sharing the latest news is one of the main objectives of digital communications. However, science appeals to people when it speaks to them. Sometimes, there is no need to find a breaking news story to have success; an amazing image can suffice. You can connect with your audience using specific opportunities (e.g. a joke on April Fools' day).

It's all about bringing people closer to science. The process is easier when they can put a face to a name and start a conversation. Social media gives you the opportunity to highlight the humanity behind the physics. In the world of science and physics, scientists are the stars, the ambassadors of their laboratories. Who more than them can convince people that science is for everyone? 
Finally, as social media platforms offer a lot of possibilities in terms of content, it's important to think about how to disseminate information: photos or videos, tone of voice, etc. Understanding and knowing the different platforms can help you choose the right message. 